\title{
Overview: Study of a Various Factors Needed for Biomedical Embedded System
}

\author{
Surabhi Joshi \\ Department of Electronics and Communication \\ Engineering \\ CTAE, MPUAT, Udaipur, India
}

\author{
Aaliya Khan \\ Department of Electronics and Communication \\ Engineering \\ CTAE, MPUAT, Udaipur, India
}

\begin{abstract}
In this paper, we describe various factors, components, technologies needed in Health Monitoring System. Nowadays, in biomedical embedded system, we have various microcontroller like Arduino, 8051 Microcontroller, Tiva C series CC3200, MSP430G2 etc but choosing which one is best is challenging because each controller has its own specification. So here this paper focuses on most of the factor that are needed in designing healthcare monitoring system.
\end{abstract}

Keywords- Biomedical sensors, Data protection, Encoding, IoT technology, Health Monitoring System, Microcontrollers

\section{INTRODUCTION}

Day by Day, the change in lifestyle increases, change in environment which causes arising in the health problem, require a daily or time to time checkup to ensure about the health. But the busy world ignoring their health issue and don't have time for their routine checkup. So this reason vanishes the traditional healthcare solution and has moved to the real time health monitoring system. These systems have the ability to provide the e-health to meet the requirement of the growing population. Before making real time system for health monitoring we have to consider various factors which are explained in next sections of the paper. Internet of Things (IoT) is a technology which enables real time data gain, data transfer, device connectivity, to control end user application. The term Internet of Medical Things (IoMT), basically used in the healthcare application which consists of connected devices that sense vital data of human physiological parameter in real time.

\section{LITERATURE SURVEY}

[1] Choudhary et al (2012) worked on the real time health monitoring using pulse-oximeter. This system includes a microcontroller interfacing with a Zigbee module which is used for communicating the data of pulse-oximeter and sending data to PC. They have used the wireless personal area network (WPAN). This system is designed for reducing cost and comfortable in our daily life usage.

[2] Pawar et al (2014) have designed effective health monitoring home base system for infant to elderly subjects. d This paper includes a measurement unit consists of heart beat sensor which is connected to Arduino UNO. This provides a digital output when finger is placed on it. It also includes a GSM so that SMS is sent to concern person when needed.

[3] Thomas et al (2016), The objective of their work is to make useful for those people who are far away from doctors and don't able to hospitals regularly. This paper consists of microcontroller Arduinomega2560 interfaced with LM35 temperature sensor and pulse sensor. Resultant output is displayed on android.

[4] Ganesan et al (2015) worked on CC3200 microcontroller interfaced with temperature sensor, pulse sensor and accelerometer. The output will be stored in the database of the server. In this work, data have been made available through web server so that hospital staffs can retrieve the information for use. The objective of their work is to make patient's data available anywhere by assigning unique identity to the patient. [5] Hussian et al (2017) implement two sensors mainly heartbeat sensor and body temperature sensor which are interfaced with Arduino board and the resultant output is shown on screen. The patient condition's data are stored and obtained using IoT technology. The objective of the project is to reduce the regular checkup and improve the quality of care in remote location.

[6] Sahu et al (2017) used the GSM technology and LabView software for analyzing the data. Two sensors like heartbeat and temperature sensor is used with microcontroller Arduino. In their proposed work, they have combined the two parameter in single device. Overall their objective is to design a device to analyze a data and give a alert to doctor using GSM900A.

[7] R.Kumar et al (2016) focused on the temperature, heartbeat, accelerometer and respiration monitoring using Raspberry pi. After connecting all sensors to board there is two ways to access the data, One is using the putty software to see the output and another one is to connect keyboard, mouse, monitor to Raspberry board and output will be shown in monitor.

In all the papers of literature survey, they have used different controllers, biomedical sensors, technologies etc. in their work according to their application but the point is we have to use only those factors which are reliable in making real time health monitoring system i.e. which controllers is best so that it consumes less power, can provide security etc. 


\section{IMPORTANT FACTORS}

\section{A. Microcontrollers}

In table number 1, we have discussed various controllers as per needed for particular applications.

As we see in the table 1 Texas Instrumentation TI CC3200, CC3220MOD provides advance security, having ARM
CortexM4 processor $(80 \mathrm{MHz})$,inbuilt Wifi Booster which are absent in maximum arduino boards but the processor of Arduino YNU provides up to $400 \mathrm{MHz}$ Clock speed and there is 4 UART in ArduinoMega2560.

Table 1 Microcontrollers and their specifications

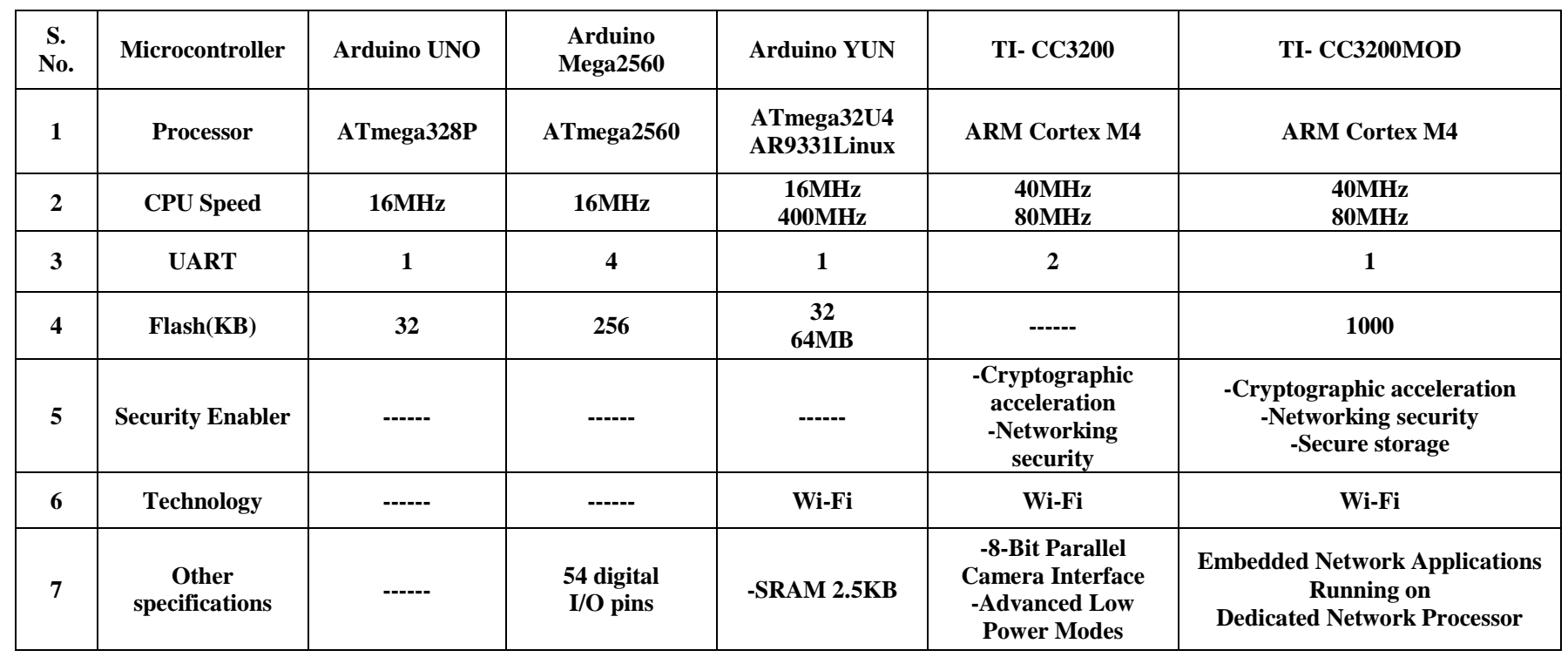

\section{B. Sensors system}

Data Acquisition happens first in the wellbeing checking condition. In this stage, different sources are utilized to accumulate the data identified with the physical status of the subject, their conduct; their condition, the exercises they have performed and so on. There are three principle classes of interconnected systems, which are frequently utilized in this domain:

(1) Personal Sensor Networks (PSN)

(2) Body Sensor Networks (BSN)

(3) Multimedia Devices (MD)

Chosen sensors and gadgets are coordinated into home articles and framework and associated utilizing of related network technologies. Each sensor is in charge of at least one undertaking in the meantime.

PSNs are utilized to identify human day by day exercises and measure conditions in the subject's condition. BSNs are utilized to screen crucial signs and wellbeing conditions by estimating physiological parameters and identifying wandering exercises. At long last, increasingly relevant data identified with human action is gathered by means of MD to screen development, ecological changes and to expand the connection between the checked subject and the e-wellbeing application. An outline of sensor organizes in Health monitoring system is portrayed in Table 2 depicts and gives the reason to the three system classifications, including the sensors and gadgets that are used in Health monitoring system.

Table 2 Health monitoring devices and sensors

\begin{tabular}{|c|c|c|c|}
\hline Category & Name & Work & Data Format \\
\hline \multirow{3}{*}{ PSN } & RFID & $\begin{array}{l}\text { Persons and objects } \\
\text { identification }\end{array}$ & Categorical \\
\hline & Temperature & Measures temperature & Time Series \\
\hline & Weight & Measures weight & Numerical \\
\hline \multirow{2}{*}{ BSN } & $\begin{array}{c}\text { Acceleromete } \\
\mathbf{r}\end{array}$ & $\begin{array}{l}\text { Measure acceleration, } \\
\text { fall detection }\end{array}$ & Time Series \\
\hline & ECG & $\begin{array}{l}\text { Monitor cardiac } \\
\text { activity }\end{array}$ & Analog Signal \\
\hline \multirow{3}{*}{ MD } & Cameras & $\begin{array}{l}\text { Monitoring and } \\
\text { tracking }\end{array}$ & $\begin{array}{l}\text { Image, } \\
\text { video }\end{array}$ \\
\hline & Microphone & Voice detection & Audio \\
\hline & Speakers & $\begin{array}{c}\text { Alerts and } \\
\text { instructions }\end{array}$ & Audio \\
\hline
\end{tabular}




\section{Physiological health parameters/sensors}

Physiological applications raised for the constant remote checking of human physiological activities. They seize and transmit essential vital signal parameters such as pulse, circulatory strain, SPO2 body temperature, and galvanic skin response and blood glucose levels. These parameters are typically utilized for further analysis and association in situations wherever abnormal conditions are detected. For this reason, the device allows healthcare companies to reveal the clinical circumstance of the subjects and straight away offer appropriate services [8]. Various biomedical sensors are as follows:

1. Body Temperature: Body temperature is easy to observe and it can depict various health issue. Based on human age, the normal temperature of a body are as follows:

- Kids $=36.6^{\circ} \mathrm{C}$ to $37.2^{\circ} \mathrm{C}$

- Adults $=36.1^{\circ} \mathrm{C}$ to $37.2^{\circ} \mathrm{C}$ ).

- Above age $65=36.2^{\circ} \mathrm{C}$

There are various body temperature sensors which are used in health monitoring system which are as follows:

Table 3 Temperature Sensors

\begin{tabular}{|c|c|c|c|}
\hline Sensor Name & Output Type & $\begin{array}{c}\text { Temperature } \\
\text { Range }\end{array}$ & Accuracy \\
\hline TMP36 & Analog & -40 to $+125{ }^{\circ} \mathrm{C}$ & $+/-1-2{ }^{\circ} \mathrm{C}$ \\
\hline LM35 & Analog & -55 to $+150{ }^{\circ} \mathrm{C}$ & $+/-0.5{ }^{\circ} \mathrm{C}$ \\
\hline DS18B20 & Digital & -55 to $+125{ }^{\circ} \mathrm{C}$ & $+/-0.5^{\circ} \mathrm{C}$ \\
\hline
\end{tabular}

2. Heart rate sensor: Heart beat sensor offers a simple manner to look at the feature of the heart. There are various heart rate sensors are available in a market which can be used in making health monitoring system or ant biomedical embedded system. One of them is pulse sensor which can measure heart rate per minute and used in [3] work. Second is LM358 is used in [9]. Infrared sensor can also be used as heart rate detector which uses the light principle.

3. ECG sensor: It is termed as ElectroCardiacGram Sensor. Most widely used ECG monitor sensor module is based on AD8232 analog IC. It has a ability to measure the electrical activity of a heart. We can extract the features of ECG signal like RR interval, QRS complex etc. using 3 lead [10] or 12 lead ECG sensor.

In a series with biomedical sensors there are lots of other sensor which are also used in health monitoring system such as galvanic skin response (used to measure skin conductance), Blood pressure sensor, SPO2 sensor (used to measure saturated oxygen), Glucose level indicator etc. So it depends on you what sensors you want to include in your work.

\section{IoT and cloud technology}

Cloud technology and internet of things, both are different technologies. IoT is related to connecting devices to internet while cloud computing includes delivering data, photos etc over a internet. The Internet of Things as basically an association between the physical and advanced universes. The advanced world communicates with the physical world utilizing a plenty of sensors and devices [11].If we talk about IoT in medical world then the term internet of medical things (IoMT) is defined as, a healthcare utility of the IoT technology, incorporates a network of connected devices that experience essential statistics in real time [12].

There are various IoT platform where we can perform cloud computing in which ThingSpeak cloud is widely used in when we talk about making prototype project, is a open source IoT application and used as cloud service provider. It provides visualization and analyzation of live stream data in a cloud. It has ability to execute MATLAB coding so we can perform online processing of data. Other than that, IBM cloud, AT \& T cloud, Amazon, IBM, Oracle, Google, cloud services can be used to perform IoT task.

Apart from excellency of cloud technology, mobile computing comes under the one of the fast growing technology in all field. Example: Blynk App, it connects your devices to cloud [13].

\section{Communication Gateway}

Network management and their interconnection is the main function of communication gateway thus it becomes the main component for real time health monitoring application. Communication gateway can be wired or wireless. Nowadays various wireless technologies are used to employ some remote system. Some of them are WiFi, Zigbee, Bluetooth etc. and which technology has to be addressed in work is still an open issue. Issue includes power consumption; cost, reliability, high speed etc. are the main requirement. Table 4 shows various wireless communication gateways are as follows:

Table 4. Communication gateway

\begin{tabular}{|c|c|c|c|}
\hline Parameter & WiFi & Zigbee & Bluetooth \\
\hline $\begin{array}{c}\text { Power } \\
\text { consumption }\end{array}$ & High & Low & Medium \\
\hline Data Rate & $11-54$ Mbs & $\begin{array}{c}\text { Approx.250 } \\
\text { Kps }\end{array}$ & 1 -3 Mbs \\
\hline Standard & IEEE 802.11 & IEEE 802.15.4 & IEEE 802.15.1 \\
\hline Topology & $\begin{array}{c}\text { Star, point to } \\
\text { point }\end{array}$ & Bus, star & Piconet \\
\hline
\end{tabular}

\section{MAJOR CHALLENGES IN HEALTH MONITORING SYSTEM}

\section{A. Security and privacy}

Without thinking about security necessities usually makes patients liable to privacy troubles when deploying wireless technology in health monitoring system[g]. The transfer of information over a internet can cause threats to patient's data. Conventional network includes:

(i) Availability

(ii) Integrity 


\section{International Journal of Engineering Applied Sciences and Technology, 2019 \\ Vol. 3, Issue 12, ISSN No. 2455-2143, Pages 59-62 \\ Published Online April 2019 in IJEAST (http://www.ijeast.com)}

(iii) Confidentiality

(iv) Access control

(v) Authentication

The security necessities in health monitoring system are same as the conventional network. Security is a critical issue in health monitoring system. Existing cryptographic methods should be used to provide patient's data protection in opposition to attacks because health monitoring system deals with sensitive data.

\section{B. Power consumption}

Energy/power consumption is also major concern along with data security. Health monitoring system should use devices, protocols of low power consumption so that it will save energy for further use. As health monitoring system uses various biosensors and devices, we should take it in concern about devices, protocols, communication gateway's power consumption choices.

\section{Accurate and continuous monitoring}

Patient like chronic disease, they always needs constant monitoring so if any abnormal activity occur, system can detect abnormality on that time.

\section{Storage capacity}

As using the IoT technologies, we have limited storage capacity to store or save patient's data. Storage capacity is also increasing day by day but instead of this, we also make sure that large amount of subject's data can be store in minimum place. Compression of the data is best option for this. It can be lossy or lossless compression of data. In medical field, lossless compression technique should be considered because medical data is very sensitive, minor change can cause many problems.

\section{CONCLUSION}

In this paper, we have discussed various aspects for the designing of health monitoring system. The important factors related to system are discussed with various challenges that are ongoing issue. Security is a major concern when we talk about sending very crucial and sensitive data via wireless communication network.

\section{REFERENCES}

[1] Choudhary, Deepak,Kumar, Rakesh and Gupta, Neeru. (2012). "Real-Time Health Monitoring System on Wireless", IJAITI, vol 1, ( pp 37-43).

[2] Pawar, Prajakta A. (2014). "Heart Rate Monitoring System using IR base Sensor \& Arduino Uno," IEEE .

[3] Thomas, Salomi S., Saraswat, Amar, Shashwat, Anurag and Bharti, Vishal. (2016). "Sensing Heart beat and Body Temperature Digitally using Arduino," IEEE International conference on Signal Processing, Communication, Power and Embedded System (SCOPES), (pp. 1721-1724).

[4] Ganesan, R., Kanimozhi, K., Prakash, R., and Chamundeeshwari, V. Vijaya. (2015). "Internet of Things (IoT) Enabled Wireless Patient Monitoring
System using CC3200," Australian Journal of Basic and Applied Science., 9(16): (pp. 275-281).

[5] Hussian, Chesti. Altaff, K. Vuha, K., Rajani, M., and Vineeth Madhu. (2017)." Smart Health Care Monitoring using Internet of Things and Android",International Journal of Advanced Research in Electronics and Communication Engineering (IJARECE), vol. 6, ( pp. 101104).

[6] Sahu, Mohan Lal and Kaushal Jigyasu Kumar. (2017). "Real time health monitoring system using Arduino and LabVIEW with GSM Technology", International Journal of Advance Engineering and Research Development (IJAERD),(pp 1-5).

[7] Kumar, R. and Rajasekaran, Dr.M.Pallikonda. (2016).” An Iot Based Patient Monitoring System Using Raspberry Pi",IEEE.

[8] Bansal, Puneet, Malik, Meenal and Kundo, Ricktam. (2018)." Smart Heart Rate Monitoring System",IEEE, PP. $1-4$.

[9] Uplenchwar, Kartikee and Vedalankar, Aditi. (2017)."'IoT Based Health Monitoring System using Raspberry $\mathrm{Pi}$ and Arduino", International Journal of Innovative Research in Computer and Communication Engineering,vol. 5, pp. 17315-17324.

[10] Yang, Zhe, Zhou, Qihao, Lei, Lei, Zheng, Kan and Xiang, Wei. (2016)." An IoT-cloud Based Wearable ECG Monitoring System for Smart Healthcare", Springer, pp. $1-11$.

[11] O. Vermesan, P. Friess, P. Guillemin. (2011). "Internet of things strategic research roadmap," Internet of Things: Global Technological and Societal Trends, vol. 1, pp. 952.

[12] Shelke, Y., Sharma, A. (2016). "Internet of Medical Things", report published by Aranca.

[13] https://blynk.io/

[14]Zhang, K., Yang, K., Liang, X., Su, Z., Shen, X., Luo, H.H. (2015) "Security and privacy for mobile healthcare networks: from a quality of protection perspective". IEEE, Wireless Commun. 22 (4). 